\title{
3D Printed Personalized External Aortic Root Model in Marfan Syndrome with Isolated Sinus of Valsalva Aneurysm Caused by a Novel Pathogenic FBN1 p.Gly1127Cys Variant
}

\author{
Jung Sun Cho ${ }^{1,+} \mathbb{C}^{\mathbb{D}}$, Joonhong Park ${ }^{2,3,+} \mathbb{\mathbb { C }}$, Jong Bum Kwon ${ }^{4}$, Dae-Won Kim ${ }^{1}$ and Mahn-Won Park ${ }^{1, *}$ \\ 1 Department of Cardiology, College of Medicine, The Catholic University of Korea, Seoul 06591, Korea; \\ tworugi@daum.net (J.S.C.); mirinesilver@catholic.ac.kr (D.-W.K.) \\ 2 Department of Laboratory Medicine, Jeonbuk National University Medical School and Hospital, \\ Jeonju 54907, Korea; miziro@jbnu.ac.kr \\ 3 Research Institute of Clinical Medicine of Jeonbuk National University-Biomedical Research Institute of \\ Jeonbuk National University Hospital, Jeonju 54907, Korea \\ 4 Department of Thoracic and Cardiovascular Surgery, College of Medicine, The Catholic University of Korea, \\ Seoul 06591, Korea; jbkwon@catholic.ac.kr \\ * Correspondence: pmw6193@catholic.ac.kr; Tel.: +82-42-220-9505 \\ + Jung Sun Cho and Joonhong Park contributed equally to this work.
}

\section{check for} updates

Citation: Cho, J.S.; Park, J.; Kwon, J.B.; Kim, D.-W.; Park, M.-W. 3D Printed Personalized External Aortic Root Model in Marfan Syndrome with Isolated Sinus of Valsalva Aneurysm Caused by a Novel Pathogenic FBN1 p.Gly1127Cys Variant. Diagnostics 2021, 11, 1057. https://doi.org/10.3390/

diagnostics11061057

Academic Editor: Nicola Martinelli

Received: 3 May 2021

Accepted: 7 June 2021

Published: 8 June 2021

Publisher's Note: MDPI stays neutral with regard to jurisdictional claims in published maps and institutional affiliations.

Copyright: (c) 2021 by the authors. Licensee MDPI, Basel, Switzerland. This article is an open access article distributed under the terms and conditions of the Creative Commons Attribution (CC BY) license (https:/ / creativecommons.org/licenses/by/ $4.0 /)$.

\begin{abstract}
The major cause of death in Marfan syndrome (MFS) is cardiovascular complications, particularly progressive dilatation of the proximal aorta, rendering these patients at risk of aortic dissection or fatal rupture. We report a 3D printed personalized external aortic root model for MFS with an isolated sinus of Valsalva aneurysm caused by a novel pathogenic FBN1 variant. A 67-yearold female with a history of lens dislocation and retinal detachment in the left eye was admitted for the evaluation of resting dyspnea several months prior. Transesophageal and transthoracic echocardiography revealed severe aortic valve regurgitation and a large left coronary sinus of Valsalva aneurysm in the proband. Sanger sequencing identified a heterozygous p.Gly1127Cys variant in the FBN1 gene; previously, a mutation at this amino acid position was described as pathogenic (p.Gly1127Ser; rs137854468). A 3D printed personalized external aortic root model based on a multidetector computed tomography scan was constructed to illustrate the location of the ostium of the left main coronary artery on the aneurysm of the left coronary artery cusp. Aortic root replacement with the Bentall procedure matched the exact shape of the 3D printed model. Creation of a 3D printed patient-specific model could be useful in facilitating the development of next-generation medical devices and resolving the risks of postoperative complications and aortic root disease.
\end{abstract}

Keywords: 3D printed personalized model; aortic root replacement; Marfan syndrome; isolated sinus of Valsalva aneurysm; FBN1 p.Gly1127Cys variant; Sanger sequencing

\section{Introduction}

Marfan syndrome (MFS, OMIM \#154700) is an autosomal dominant systemic disorder that affects connective tissue, primarily in the skeletal (long limbs and fingers, scoliosis, and pectus deformities), ocular (ectopia lentis), and cardiovascular (aortic aneurysm and dissection) systems. MFS is caused by the fibrillin1 gene (FBN1), which is the primary disease-associated gene of MFS [1]. In approximately three-quarters of individuals with MFS, there is an affected parent. In a quarter of individuals, MFS is due to de novo pathogenic FBN1 variants, with no differences according to sex, ethnicity, or geographical location [2]. Clinical manifestations of MFS are highly variable with a broad range of overlapping phenotypes, even among members of the same family [3]. Up to $97 \%$ of MFS patients who fulfilled the revised Ghent criteria for clinical diagnosis have FBN1 mutations encoding a major component of the extracellular matrix microfibril, namely, fibrillin-1 [4]. There are at least 3000 described pathogenic variants of FBN1, and the 
disease phenotype depends on the type of mutation involved and its effect on the fibrillin-1 protein [5]. Dominant-negative (DN) missense mutations can lead to abnormal fibrillin-1 function that results from structurally altered fibrillin, which is incorporated together with normal fibrillin-1 derived from the nonmutated allele [3]. Mortality and morbidity are associated, above all, with aortic involvement including dilation, dissection, or rupture [6] Skeletal, aortic, and pulmonary complications are more prevalent in adults with MFS than in children. Additionally, adult males are more likely to develop aortic root dilatation than females, and they more often need prophylactic aortic replacement [7]. Particularly, among MFS with a typical aortic root aneurysm, standard surgical management of aortic root aneurysm in MFS is considered to be either total root replacement or valve-sparing root replacement [8].

In this report, we described a Korean patient in which a novel p.Gly1127Cys variant in FBN1 cosegregates with isolated left coronary sinus of Valsalva aneurysm and additional manifestations of lens dislocation with minor skeletal involvement. Furthermore, we applied a three-dimensional (3D) printed personalized external aortic root model to understand abnormal aortic structure in a patient with MFS.

\section{Case Presentation}

A 67-year-old female was admitted to the Department of Cardiology, Daejeon St. Mary's Hospital (Daejeon, Korea) for the evaluation of resting dyspnea several months prior. She grew up in an orphanage when she was young and had no information about her parents. During childbirth, she developed a lens dislocation in her left eye. Lens removal surgery was performed but caused retinal detachment that led to blindness in the left eye after several ophthalmic surgeries. She had glaucoma in the right eye. She was $158 \mathrm{~cm}$ tall, but she had a slender build and arachnodactyly and a positive thumb sign. Transesophageal echocardiography (TEE) and transthoracic echocardiography (TTE) were performed to evaluate dyspnea and revealed severe aortic valve regurgitation (AR) and a large left coronary sinus of Valsalva aneurysm in the proband (Figure 1A-C). The left ventricular end-diastolic and left ventricular end-systolic dimensions were 57 and $41 \mathrm{~mm}$, respectively. The left ventricular ejection fraction was 56\%. Multi-detector computed tomography (MDCT) demonstrated that the maximal diameter of the sinus of Valsalva aneurysm was $64 \mathrm{~mm}$ and that the coronary artery was patent in the proband.

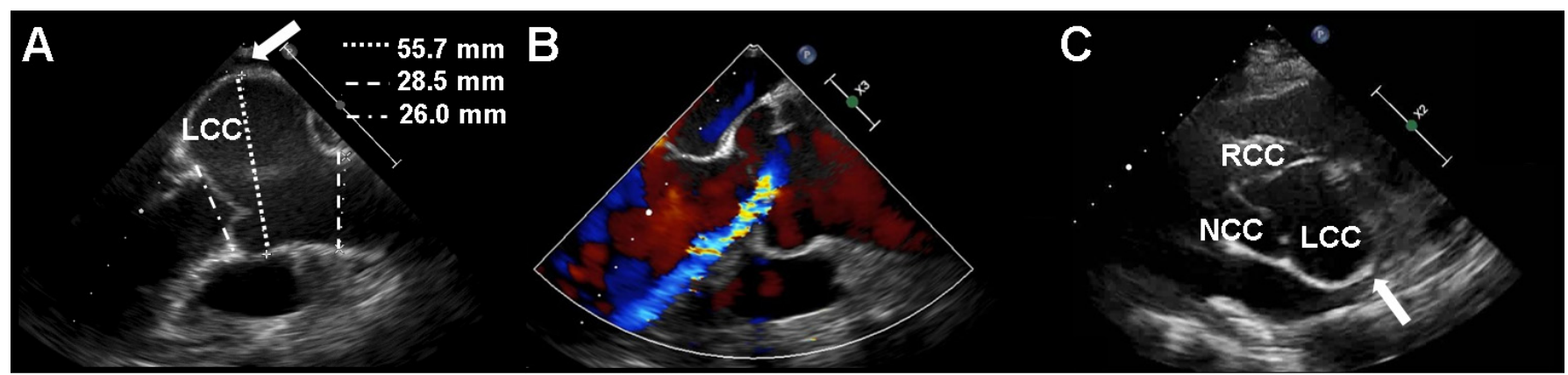

Figure 1. Transesophageal echocardiography (TEE) and transthoracic echocardiography (TTE) in the proband with Marfan syndrome. TEE (A) and (B) and TTE (C) revealed an isolated sinus of Valsalva aneurysm of the left coronary cusp (arrow) with severe aortic regurgitation. LCC, left coronary cusp; RCC, right coronary cusp; NCC, noncoronary cusp.

The proband was going to undergo aortic root replacement (ARR) with the Bentall procedure due to the isolated left coronary sinus of Valsalva aneurysm and severe AR. To help understand the anatomic structure before the Bentall procedure, a 3D printed personalized external aortic root model based on the MDCT scan was constructed to illustrate the location of the ostium of the left main coronary artery on the aneurysm of the left coronary artery cusp. The patient's CT datasets were imported into a medical 3D image-based engineering software (Materialize Mimics ver. 22 and 3-Matic ver. 13, 
Materialise NV, Leuven, Belgium) and the 3D model was printed on a Stratasys Connex3 Objet500 3D printer (Stratasys Ltd., Eden Prairie, MN, USA) using the form2 clear resin (FLGPCL02). At first, we considered valve sparing ARR for this case because aortic leaflets were relatively preserved even though annular dilatation was combined $(26 \mathrm{~mm})$. However, 3D printed model revealed that thinning of the cusp due to asymmetrically severely enlarged left coronary cusp can cause stress fenestration toward the commissures and turbulent blood flow inside the aorta (Figure 2A-D). Proband's surgical anatomic model and 3D printed model were exactly matched (Figure 2E). Finally, she underwent a modified Bentall operation with a $21 \mathrm{~mm}$ mechanical aortic valve and left and right coronary artery buttons successfully.

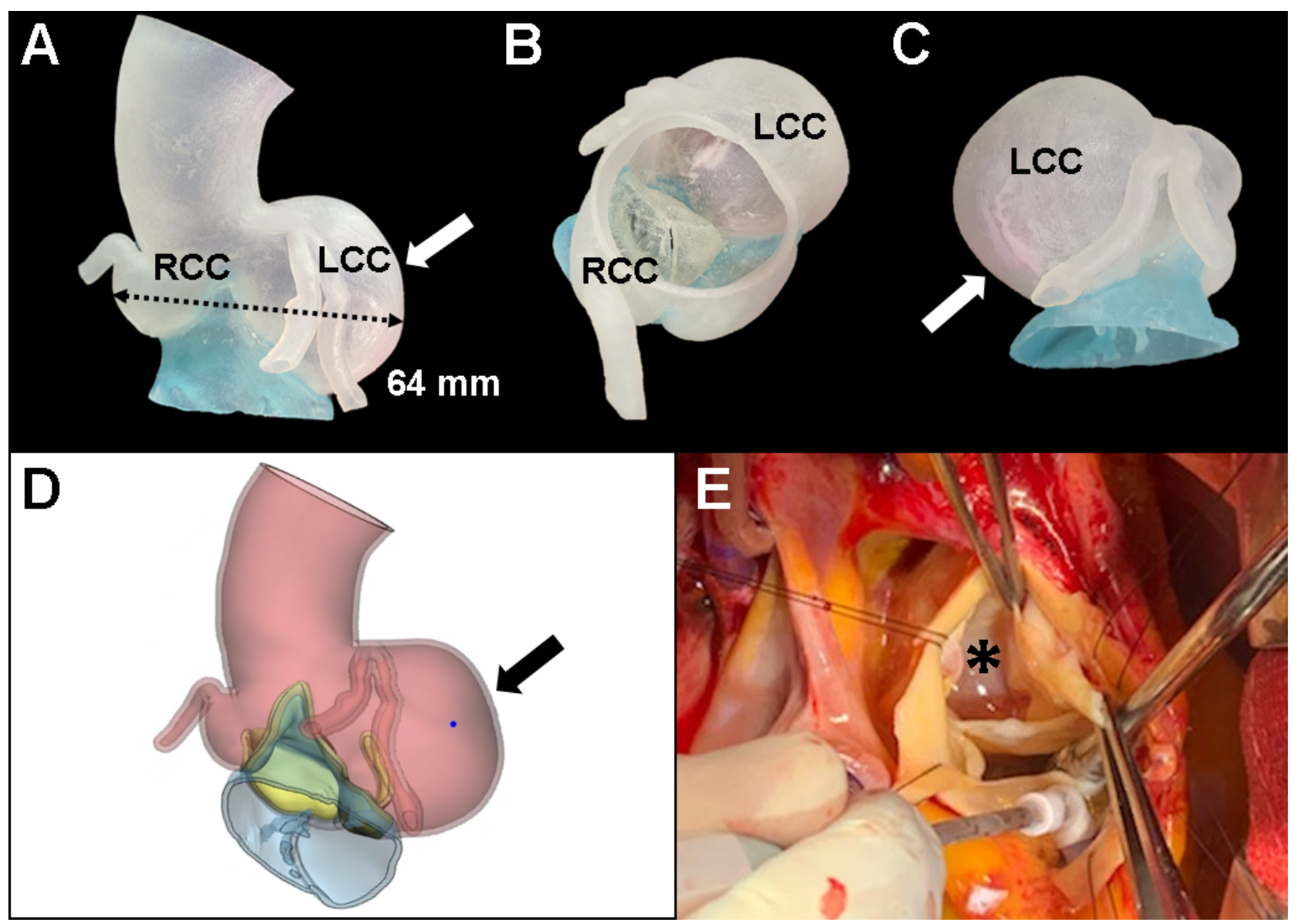

Figure 2. 3D printed model in the proband with Marfan syndrome. To help understand the anatomic structure before surgery, a 3D printed personalized external aortic root model (A-D) was derived from multi-detector computed tomography to illustrate the location of the ostium of the left main aneurysm of the left coronary cusp (arrow). (E) Aortic root replacement with the Bentall procedure matched the exact shape of the 3D printed model (asterisk). LCC, left coronary cusp; RCC, right coronary cusp; NCC, noncoronary cusp.

To identify the potential genetic cause of MFS in the proband (II-1 in Figure 3A), Sanger sequencing was recommended. Genomic DNA was extracted from peripheral blood using a QIAamp DNA Mini Kit (Qiagen GmbH, Hilden, Germany). Entire coding exons and flanking intronic sequences of the FBN1 gene were amplified by polymerase chain reaction using the primers described previously [9]. Sanger sequencing of PCR products was performed using the BigDye Terminator v3.1 Cycle Sequencing Kit (Applied Biosystems, Foster City, CA, USA) and was resolved by capillary electrophoresis on an 3730XL Genetic Analyzer (Applied Biosystems, Carlsbad, CA, USA). Electropherograms were interpreted using Sequencher DNA Sequence Analysis Software Ver 4.9 (Gene Codes Corporation, Ann Arbor, MI, USA). All identified variants were confirmed by bidirectional resequencing. The FBN1 sequence with RefSeq ID NM_000138.4 was used as a reference for cDNA nucleotide numbering. Mutations predicted to cause strong and moderate alterations in 
gene functions were manually reviewed by laboratory geneticists based on ACMG-AMP standards and guidelines [10]. As a result, Sanger sequencing identified a heterozygous c.3379G $>$ T mutation, resulting in an amino acid change of glycine to cysteine at position 1127 (NM_000138.4: c.3379G>T; p.Gly1127Cys) in the FBN1 gene (Figure 3B). Another mutation at the same amino acid position had previously been described as pathogenic (NM_000138.4: c.3379G>A; p.Gly1127Ser; rs137854468) [11]. Sequence alignment of the conserved calcium binding epidermal growth factor (cbEGF)-like domain of the FBN1 protein demonstrated that the Gly1127 residue is highly conserved across multiple vertebrate species (Figure 3C).

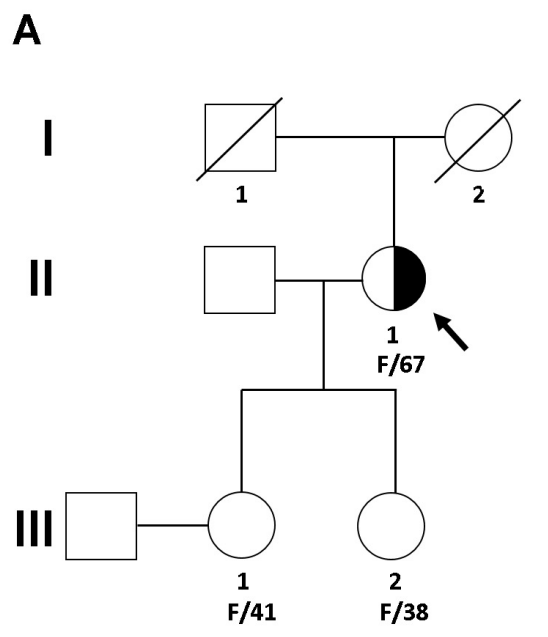

B
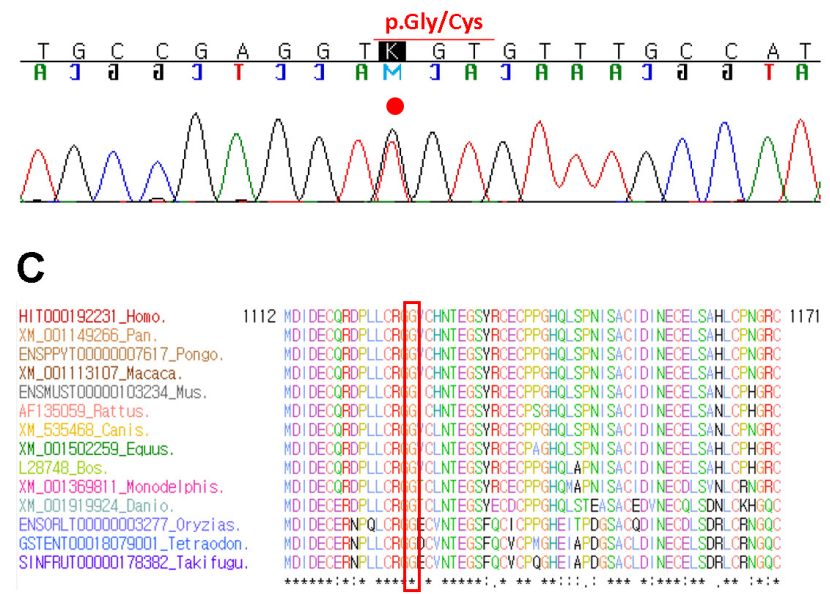

Figure 3. Pedigree analysis and sequencing results. (A) Pedigree of the proband (arrow) with Marfan syndrome caused by a heterozygous FBN1 variant and her healthy family members. (B) Sanger sequencing revealed a heterozygous missense variant (NM_000138.4: c.3379G>T; p.Gly1127Cys) of the FBN1 gene in the proband. The variant is highlighted by the red dot. (C) Sequence alignment of the conserved calcium binding epidermal growth factor (cbEGF)-like domain of the FBN1 protein in multiple vertebrate species. The protein sequence of the Gly1127 residue is highly conserved across the compared vertebrate species. It is highlighted in the red box.

In approximately $75 \%$ of individuals with MFS, the syndrome is inherited in an autosomal dominant manner from an affected parent [12]. Thus, genetic counseling and segregation analysis were recommended for the family members of the proband. She had two daughters aged 41 and 38, and no MFS clinical symptoms were found during their echocardiography or physical examination. They refused consent for the genetic test, and genetic counseling and segregation analysis were not evaluated.

\section{Discussion}

Mutated fibrillin-1 results in dysregulation of TGF- $\beta$ signaling as well as structural weakness of connective tissue, both of which contribute to complicated pathogenesis in MFS [13]. It plays a communicative role in biosignaling by regulating the local bioavailability of TGF- $\beta$, and it plays a role in mechanosignaling by interacting with mechanosensors and providing feedback to regulate the response to hemodynamic changes [14]. FBN1 mutations have been associated with a broad spectrum of phenotypes in classic MFS, and while some correlations between the FBN1 genotype and clinical phenotype are known, few are predictive [15]. The correlation between FBN1 genotypes and phenotypes has been extensively reported, and more than 3000 pathogenic mutations are distributed throughout the entire length of the gene $[3,5,15,16]$. FBN1 variants with cysteine (Cys) substitutions are more frequently associated with a high prevalence of ectopia lentis such as partial or complete displacement of the eye lens [17]. FBN1 mutations in exons 24-32 define a high-risk group for cardiovascular manifestations at all ages [15]. MFS with haploinsufficient type $F B N 1$ variants such as nonsense and out-of-frame variants that presumably 
cause nonsense-mediated mRNA decay have more severe aortic phenotypes than those with DN-type mutations such as missense and in-frame variants that are expected to exert loss-of-function effects $[3,5,16,17]$. Deleterious variants among DN patients showed that patients with mutations affecting or creating cysteine residues and in-frame deletion variants in the cbEGF domains of exons 25-36 and 43-49 (DN-CD variants) had a 6.3-fold higher risk for aortic events than DN-nonCD patients, which is comparable to or more deleterious than haploinsufficient variants [3]. In our case, a novel missense p.Gly1127Cys mutation was identified at the same position in the FBN1 gene as others that have caused different substitutions of the Gly1127 residue. The proband had complete displacement of the left eye lens during childbirth, and subsequent retinal detachment and total blindness developed. She had glaucoma in the right eye. She suffered from all kinds of ocular complications of MFS. The pathogenic criterion for this novel variant is weighted as moderate 5 (PM5) based on ACMG-AMP standards and guidelines [10]. A novel missense amino acid change occurring at the same position as another pathogenic missense change is considered moderate evidence but cannot be assumed to be pathogenic. This is especially true if the novel change is more conservative than the established pathogenic missense variant. Additionally, the different amino acid changes could lead to a different phenotype [10]. Francke and colleagues [11] reported that a Gly1127Ser mutation in the cbEGF-like domain of the FBN1 gene produces a mild form of autosomal dominantly inherited weakness of elastic tissue, which predisposes patients to ascending aortic aneurysm and dissection later in life. Additionally, in our case, the proband had an isolated left coronary sinus of Valsalva aneurysm without dissection in late life. Recently, Hernándiz A and colleagues [18] reported evidence of the different clinical behaviors of patients with either a DN effect or haploinsufficiency of the FBN1 variants and of males versus females. In addition, the observation of a "Carter effect" in the cardiovascular phenotype provides further evidence of the existence of inherited modifiers and supports the high heritability of cardiovascular clinical features [19].

The major cause of death in MFS is cardiovascular complications, particularly progressive dilatation of the proximal aorta, rendering these patients at risk of aortic dissection or fatal rupture [20]. Although the aortic sinus is most commonly affected, aneurysms and dissections in more distal aortic regions and in extra-aortic arteries can also occur [21]. The progression of pulmonary artery dilatation is associated with the occurrence of type B dissection, which might reflect tissue vulnerability [22]. Current standard management is echocardiographic monitoring with a consideration to replace the ascending aorta, with or without aortic valve replacement. Prophylactic root replacement potentially neutralizes that hazard and is the recommended management for patients who meet the widely accepted criteria defining them as high risk [14]. Personalized external aortic root support is computer designed and manufactured to match the aortic root morphology of the individual patient based on multiple measurements of images before and after surgery. This approach is intended to prevent ongoing dilatation of an already morphologically abnormal aorta with the purpose of reducing the risk of death due to dissection in the aortic root [23]. The operation does not require opening the heart or aorta or any interference with cerebral or myocardial perfusion, and it is usually performed without the need for cardiopulmonary bypass. It is applicable to patients with root aneurysms caused by genetically determined etiologies such as MFS and Loeys-Dietz and Ehlers-Danlos syndrome [8]. Isolated left coronary sinuses of Valsalva aneurysms are extremely rare cardiovascular manifestations in patients with MFS, and few cases have been reported [24,25]. Aortic root $3 \mathrm{D}$ printing is an emerging technique and may improve the success rate and safety of aortic valve replacement [26,27]. Visualization via 3D printing can help surgeons and cardiologists understand the anatomy of rare aortic root disease and plan the procedure. In this case, we applied 3D printing, and the surgical findings of the proband matched the 3D printed model such as the location of the ostium of the left main coronary artery on the aneurysm of the left coronary artery cusp. Furthermore, patient-specific 3D printing models such as this case are expected to help surgeons reduce surgery time, side effects, 
and complications [26,27]. In our case, severe AR with aortic root aneurysm could be performed composite valve graft or valve sparing aortic root replacement. Valve sparing aortic root replacement can avoid anticoagulation and prosthesis-related complications. However, a composite valve graft was performed as a modified Bentall operation after analysis of the 3D model because of aortic annular dilatation (aortic annulus, $26 \mathrm{~mm}$ ) and thinning of the cusps due to the asymmetrically severely enlarged left coronary cusp. Thinning of the cusps when involved with more than one cusp, valve sparing aortic root replacement is not advised. Additionally, more than $25 \mathrm{~mm}$ annular dilatation was a risk factor of early and late failure [28].

\section{Conclusions}

In conclusion, we reported a 3D printed personalized external aortic root model in MFS with an isolated left coronary sinus of Valsalva aneurysm caused by a novel pathogenic FBN1 p.Gly1127Cys variant. Creation of a 3D printed patient-specific model could be useful in facilitating the development of next-generation medical devices and resolving the risks of postoperative complications and aortic root disease. Genetic analysis of the FBN1 gene is essential to discover its genetic origin in MFS patients with isolated left coronary sinus sinuses of Valsalva aneurysm.

Author Contributions: J.S.C. collected clinical medical records and drafted the manuscript. J.P. interpreted the sequencing data and drafted the manuscript. J.B.K. performed the surgical procedure. D.-W.K. collected clinical medical records. M.-W.P. interpreted the clinical medical records and contributed to the conception and design of the study. All authors have read and agreed to the published version of the manuscript.

Funding: The authors wish to acknowledge the financial support of the Catholic Medical Center Research Foundation made in 2021 (grant number: 52021B000100008).

Institutional Review Board Statement: This study protocol was approved by the Institutional Review Board of the Catholic University of Korea (Approval number: DC21ZASE0012; Date of approval: 9 March 2021).

Informed Consent Statement: All subjects provided written informed consent for clinical and molecular analyses and publication before the study.

Data Availability Statement: The data presented in this study are available upon request from the corresponding author.

Conflicts of Interest: The authors declare no conflict of interest.

\section{References}

1. Groth, K.A.; Hove, H.; Kyhl, K.; Folkestad, L.; Gaustadnes, M.; Vejlstrup, N.; Stochholm, K.; Østergaard, J.R.; Andersen, N.H.; Gravholt, C.H. Prevalence, incidence, and age at diagnosis in Marfan Syndrome. Orphanet. J. Rare Dis. 2015, 10, 153. [CrossRef]

2. Sakai, L.Y.; Keene, D.R.; Renard, M.; De Backer, J. FBN1: The disease-causing gene for Marfan syndrome and other genetic disorders. Gene 2016, 591, 279-291. [CrossRef]

3. Takeda, N.; Inuzuka, R.; Maemura, S.; Morita, H.; Nawata, K.; Fujita, D.; Taniguchi, Y.; Yamauchi, H.; Yagi, H.; Kato, M.; et al. Impact of Pathogenic FBN1 Variant Types on the Progression of Aortic Disease in Patients With Marfan Syndrome. Circ. Genom. Precis Med. 2018, 11, e002058. [CrossRef] [PubMed]

4. von Kodolitsch, Y.; De Backer, J.; Schüler, H.; Bannas, P.; Behzadi, C.; Bernhardt, A.M.; Hillebrand, M.; Fuisting, B.; Sheikhzadeh, S.; Rybczynski, M.; et al. Perspectives on the revised Ghent criteria for the diagnosis of Marfan syndrome. Appl. Clin. Genet. 2015, 8, 137-155. [CrossRef] [PubMed]

5. Franken, R.; Groenink, M.; de Waard, V.; Feenstra, H.M.; Scholte, A.J.; van den Berg, M.P.; Pals, G.; Zwinderman, A.H.; Timmermans, J.; Mulder, B.J. Genotype impacts survival in Marfan syndrome. Eur. Heart J. 2016, 37, 3285-3290. [CrossRef]

6. Groth, K.A.; Stochholm, K.; Hove, H.; Andersen, N.H.; Gravholt, C.H. Causes of Mortality in the Marfan Syndrome(from a Nationwide Register Study). Am. J. Cardiol. 2018, 122, 1231-1235. [CrossRef] [PubMed]

7. Roman, M.J.; Devereux, R.B.; Preiss, L.R.; Asch, F.M.; Eagle, K.A.; Holmes, K.W.; LeMaire, S.A.; Maslen, C.L.; Milewicz, D.M.; Morris, S.A.; et al. Associations of Age and Sex With Marfan Phenotype: The National Heart, Lung, and Blood Institute GenTAC (Genetically Triggered Thoracic Aortic Aneurysms and Cardiovascular Conditions) Registry. Circ. Cardiovasc. Genet. 2017, 10. [CrossRef] 
8. Treasure, T.; Takkenberg, J.J.; Golesworthy, T.; Rega, F.; Petrou, M.; Rosendahl, U.; Mohiaddin, R.; Rubens, M.; Thornton, W.; Lees, B.; et al. Personalised external aortic root support (PEARS) in Marfan syndrome: Analysis of 1-9 year outcomes by intention-to-treat in a cohort of the first 30 consecutive patients to receive a novel tissue and valve-conserving procedure, compared with the published results of aortic root replacement. Heart 2014, 100, 969-975. [CrossRef]

9. Zhao, F.; Pan, X.; Zhao, K.; Zhao, C. Two novel mutations of fibrillin-1 gene correlate with different phenotypes of Marfan syndrome in Chinese families. Mol. Vis. 2013, 19, 751-758. [PubMed]

10. Richards, S.; Aziz, N.; Bale, S.; Bick, D.; Das, S.; Gastier-Foster, J.; Grody, W.W.; Hegde, M.; Lyon, E.; Spector, E.; et al. Standards and guidelines for the interpretation of sequence variants: A joint consensus recommendation of the American College of Medical Genetics and Genomics and the Association for Molecular Pathology. Genet. Med. 2015, 17, 405-424. [CrossRef] [PubMed]

11. Francke, U.; Berg, M.A.; Tynan, K.; Brenn, T.; Liu, W.; Aoyama, T.; Gasner, C.; Miller, D.C.; Furthmayr, H. A Gly1127Ser mutation in an EGF-like domain of the fibrillin-1 gene is a risk factor for ascending aortic aneurysm and dissection. Am. J. Hum. Genet. 1995, 56, 1287-1296.

12. von Kodolitsch, Y.; Robinson, P.N. Marfan syndrome: An update of genetics, medical and surgical management. Heart 2007, 93, 755-760. [CrossRef] [PubMed]

13. Takeda, N.; Hara, H.; Fujiwara, T.; Kanaya, T.; Maemura, S.; Komuro, I. TGF- $\beta$ Signaling-Related Genes and Thoracic Aortic Aneurysms and Dissections. Int J. Mol. Sci 2018, 19, 2125. [CrossRef] [PubMed]

14. Ramirez, F.; Caescu, C.; Wondimu, E.; Galatioto, J. Marfan syndrome; A connective tissue disease at the crossroads of mechanotransduction, TGF $\beta$ signaling and cell stemness. Matrix Biol. 2018, 71-72, 82-89. [CrossRef] [PubMed]

15. Faivre, L.; Collod-Beroud, G.; Loeys, B.L.; Child, A.; Binquet, C.; Gautier, E.; Callewaert, B.; Arbustini, E.; Mayer, K.; ArslanKirchner, M.; et al. Effect of mutation type and location on clinical outcome in 1013 probands with Marfan syndrome or related phenotypes and FBN1 mutations: An international study. Am. J. Hum. Genet. 2007, 81, 454-466. [CrossRef] [PubMed]

16. Franken, R.; Teixido-Tura, G.; Brion, M.; Forteza, A.; Rodriguez-Palomares, J.; Gutierrez, L.; Garcia Dorado, D.; Pals, G.; Mulder, B.J.; Evangelista, A. Relationship between fibrillin-1 genotype and severity of cardiovascular involvement in Marfan syndrome. Heart 2017, 103, 1795-1799. [CrossRef]

17. Schrijver, I.; Liu, W.; Odom, R.; Brenn, T.; Oefner, P.; Furthmayr, H.; Francke, U. Premature termination mutations in FBN1: Distinct effects on differential allelic expression and on protein and clinical phenotypes. Am. J. Hum. Genet. 2002, 71, $223-237$. [CrossRef]

18. Hernándiz, A.; Zúñiga, A.; Valera, F.; Domingo, D.; Ontoria-Oviedo, I.; Marí, J.F.; Román, J.A.; Calvo, I.; Insa, B.; Gómez, R.; et al. Genotype FBN1/phenotype relationship in a cohort of patients with Marfan syndrome. Clin. Genet. 2021, 99, 269-280. [CrossRef]

19. Grange, T.; Aubart, M.; Langeois, M.; Benarroch, L.; Arnaud, P.; Milleron, O.; Eliahou, L.; Gross, M.S.; Hanna, N.; Boileau, C.; et al. Quantifying the Genetic Basis of Marfan Syndrome Clinical Variability. Genes (Basel) 2020, 11, 574. [CrossRef]

20. Vanem, T.T.; Geiran, O.R.; Krohg-Sørensen, K.; Røe, C.; Paus, B.; Rand-Hendriksen, S. Survival, causes of death, and cardiovascular events in patients with Marfan syndrome. Mol. Genet. Genom. Med. 2018, 6, 1114-1123. [CrossRef]

21. Demolder, A.; von Kodolitsch, Y.; Muiño-Mosquera, L.; De Backer, J. Myocardial Function, Heart Failure and Arrhythmia in Marfan Syndrome: A Systematic Literature Review. Diagnostics (Basel) 2020, 10, 751. [CrossRef] [PubMed]

22. Brouwer, C.; Bulut, H.; van Gemert, W.; Staal, A.H.; Cortenbach, K.; Snoeren, M.; Nijveldt, R.; Duijnhouwer, A.; Loeys, B.L.; van Royen, N.; et al. Progressive Pulmonary Artery Dilatation is Associated with Type B Aortic Dissection in Patients with Marfan Syndrome. J. Clin. Med. 2019, 8, 1848. [CrossRef]

23. Treasure, T.; Pepper, J. Personalised External Aortic Root Support (PEARS) Compared with Alternatives for People with Life-Threatening Genetically Determined Aneurysms of the Aortic Root. Diseases 2015, 3, 2-14. [CrossRef] [PubMed]

24. Zhang, M.; Zhou, Y.; Peng, Y.; Jin, L. Two rare missense mutations in the fibrillin-1 gene associated with atypical cardiovascular manifestations in a Chinese patient affected by Marfan syndrome. Mol. Med. Rep. 2018, 18, 877-881. [CrossRef] [PubMed]

25. Chamsi-Pasha, M.A.; Lawrie, G.M. Aneurysmal left sinus of Valsalva in Marfan's syndrome. Eur. Heart J. 2018, 39, 285. [CrossRef] [PubMed]

26. Oliveira-Santos, M.; Oliveira-Santos, E.; Gonçalves, L.; Silva Marques, J. Cardiovascular Three-Dimensional Printing in NonCongenital Percutaneous Interventions. Heart Lung Circ. 2019, 28, 1525-1534. [CrossRef]

27. Haghiashtiani, G.; Qiu, K.; Zhingre Sanchez, J.D.; Fuenning, Z.J.; Nair, P.; Ahlberg, S.E.; Iaizzo, P.A.; McAlpine, M.C. 3D printed patient-specific aortic root models with internal sensors for minimally invasive applications. Sci. Adv. 2020, 6, eabb4641. [CrossRef] [PubMed]

28. Koolbergen, D.R.; Manshanden, J.S.; Bouma, B.J.; Blom, N.A.; Mulder, B.J.; de Mol, B.A.; Hazekamp, M.G. Valve-sparing aortic root replacement+. Eur. J. Cardiothorac. Surg. 2015, 47, 348-354; discussion 354. [CrossRef] 